\title{
Lattice-matched HfN buffer layers for epitaxy of GaN on Si
}

R. Armitage ${ }^{\text {a) }}$, Qing Yang, H. Feick ${ }^{\text {b) }}$, J. Gebauer, and E.R. Weber

Materials Sciences Division, Lawrence Berkeley National Laboratory, and Department of Materials Science and Engineering, University of California at Berkeley, California 94720

Satoko Shinkai and Katsutaka Sasaki

Department of Materials Science, Faculty of Engineering, Kitami Institute of Technology, Kitami 090-8507, Japan

\section{ABSTRACT}

Gallium nitride is grown by plasma-assisted molecular-beam epitaxy on (111) and (001) silicon substrates using sputter-deposited hafnium nitride buffer layers. Wurtzite GaN epitaxial layers are obtained on both the (111) and (001) HfN/Si surfaces, with crack-free thickness up to $1.2 \mu \mathrm{m}$. Initial results for GaN grown on the (111) surface show a photoluminescence peak width of $17 \mathrm{meV}$ at $11 \mathrm{~K}$, and an asymmetric x-ray rocking curve width of 20 arcmin. Wurtzite $\mathrm{GaN}$ on $\mathrm{HfN} / \mathrm{Si}(001)$ shows reduced structural quality and peculiar low-temperature luminescence features. However, growth on the (001) surface results in nearly stress-free films, suggesting that much thicker crack-free layers could be obtained.

\footnotetext{
a) Electronic mail: RDArmitage@lbl.gov

b) On leave from center of advanced european studies and research (caesar), Bonn, Germany
} 
Epitaxy of $\mathrm{GaN}$ on silicon offers a considerable cost advantage relative to growth on sapphire or $\mathrm{SiC}$ and the potential for monolithic integration of GaN-based devices with conventional microelectronics. However, Si substrates present additional challenges for $\mathrm{GaN}$ growth. Thick $(>1 \mu \mathrm{m}) \mathrm{GaN}$ epilayers often crack upon cooling to room temperature due to the severe tensile stress induced by the $\sim 35 \%$ smaller thermal expansion coefficient of Si. Additionally, gallium exhibits poor wetting on the Si surface and exposed regions are converted to amorphous $\mathrm{SiN}_{\mathrm{x}}$, disrupting epitaxy ${ }^{1}$. Therefore low-temperature GaN (as typically employed on sapphire) is not an effective buffer layer for Si substrates and other materials must be considered. Buffer materials investigated include $\mathrm{AlN},{ }^{1-8} \mathrm{SiC},{ }^{8,9} \mathrm{AlAs},{ }^{7,10}$ intentionally formed $\mathrm{SiN}_{\mathrm{x}},{ }^{11}$ and $\mathrm{BP} .{ }^{12}$ The best results by far have been achieved with the AlN buffer layer process, leading to the demonstration of high-brightness blue light-emitting diodes on $\mathrm{Si}^{13,14,15}$ However, the mutual solubility of $\mathrm{Si}$ and $\mathrm{Al}$ is high at the buffer layer temperature (eutectic point $\left.577^{\circ} \mathrm{C}\right)$. Therefore AIN may exacerbate interdiffusion at the interface ${ }^{6,16}$ which results in high unintentional doping levels in both the film and substrate. These drawbacks merit further investigation of alternative buffer layers.

In this letter, we show that hafnium nitride grown by magnetron sputter epitaxy is an effective buffer layer for $\mathrm{GaN}$ on $\mathrm{Si}$, with epilayer quality comparable to typical results achieved using AlN buffer layers. Hafnium nitride has the NaCl-type structure, a lattice constant of $0.452 \mathrm{~nm}$, and a mismatch of only $+0.35 \%$ for $\mathrm{GaN}(0001) / \mathrm{HfN}(111)$. It is a refractory metal and has been studied as a diffusion barrier in Si technology. Due to the small $(0.02 \%)$ mismatch for the coincident-site lattice involving $6 \mathrm{HfN}$ unit cells and 5 Si unit cells, HfN can be grown epitaxially on $\mathrm{Si}^{17}$ Like AlN, HfN is stable with regard 
to formation of $\mathrm{Si}_{3} \mathrm{~N}_{4}$, but should offer better diffusion resistance. Additionally, $\mathrm{HfN}$ is expected to form an ohmic contact to $n-G a N .{ }^{18}$ Thus its metallic conductivity could make possible novel $\mathrm{GaN}$ devices such as metal base transistors or resonant tunneling devices, and provide a built-in $\mathrm{n}$-type contact to facilitate processing of conventional devices.

Epitaxial hafnium nitride films of $200 \mathrm{~nm}$ thickness were deposited by reactive dc magnetron sputtering on $\operatorname{Si}(111)$ and (001) substrates using the process of Shinkai and Sasaki. ${ }^{17}$ It is emphasized that this process was not originally intended to prepare buffer layers for $\mathrm{GaN}$ growth, and the $450^{\circ} \mathrm{C} \mathrm{HfN}$ deposition temperature was the practical maximum for the deposition chamber rather than the optimum for HfN epitaxy. The HfN stoichiometry was confirmed by $\mathrm{x}$-ray photoelectron spectroscopy and the cube-on-cube epitaxial relationship was verified by x-ray diffraction. Rocking curves for the HfN films were broad ( $\sim 1 \mathrm{deg}$ ), and a lateral grain size of $\sim 50 \mathrm{~nm}$ was determined by scanning tunneling microscopy. Improved structural quality would be expected using a higher HfN growth temperature. Despite the limitations of the present HfN films, they yielded encouraging results as buffer layers for GaN.

Subsequent GaN growth on the HfN templates was performed by molecular-beam epitaxy (MBE) using a Ga effusion cell and an Applied Epi rf nitrogen plasma source. Plasma operating conditions were $225 \mathrm{~W}, 1.5 \mathrm{sccm} \mathrm{N}_{2}$ flow and chamber pressure of $5 \times 10^{-5}$ torr, giving a nitrogen-limited growth rate of $0.6 \mu \mathrm{m} /$ hour. The base pressure excluding hydrogen was $5 \square 10^{-10}$ torr. The substrate temperature was estimated by pyrometry, taking the $\mathrm{HfN}$ emissivity as 0.15 . Prior to initiating growth the substrates were exposed to nitrogen plasma for $5 \mathrm{~min}$. A $5 \mathrm{~nm} \mathrm{GaN}$ layer was deposited at $500^{\circ} \mathrm{C}$, 
and the temperature increased to $740^{\circ} \mathrm{C}$ over a 2 minute interval without growth interruption. Finally 1.0-1.5 $\mu \mathrm{m}$ thick nominally undoped GaN films were grown.

Mirror-like surfaces were observed for GaN on both (111) and (001)HfN/Si substrates. Atomic force microscopy showed a rms roughness of $10 \mathrm{~nm}$ and morphology similar to that of optimized MBE-GaN grown on sapphire. In this study, the maximum crack-free layer thickness was $1.0 \mu \mathrm{m}$ on $\mathrm{Si}(111)$ and at least $1.2 \mu \mathrm{m}$ on $\mathrm{Si}(001)$. Although thicker $(1.5 \mu \mathrm{m})$ films on $\mathrm{Si}(111)$ contained cracks, even these films had intact regions with areas up to several $\mathrm{mm}^{2}$.

Typical (0002) and (10-11) double-crystal x-ray rocking curve widths for GaN on HfN/Si(111) were 19 and 20 arcmin, respectively. Typical (0002) rocking curve widths for optimized GaN on AlN/Si(111) range from 8-16 arcmin, ${ }^{1,2,5,6,7}$ although a value of 14 arcsec was reported for a process employing AlGaN/GaN superlattices. ${ }^{3}$ For GaN-onsapphire, it is recognized that the asymmetric rocking curve actually shows better correlation with the threading dislocation density. ${ }^{19}$ Asymmetric rocking curve widths of 10 arcmin for the (10-12) reflection $^{15}$ and 13 arcmin for the (20-21) reflection ${ }^{6}$ were reported for $\mathrm{GaN}$ on $\mathrm{AlN} / \mathrm{Si}(111)$ grown by metal-organic vapor phase epitaxy (MOVPE). The larger width of 20 arcmin achieved on HfN(111) might be attributed to the small grain size of the buffer layer. On the other hand, MOVPE generally yields better asymmetric rocking curves than MBE, and a fair evaluation of the HfN buffer layer should involve GaN films grown by the same technique with similar substrate temperatures.

Photoluminescence spectra for an intact region of a cracked $1.5 \mu \mathrm{m} \mathrm{GaN} \mathrm{layer} \mathrm{on}$ HfN (111) are shown in Fig. 1. The excitation wavelength was $325 \mathrm{~nm}$ with an optical 
power of $\sim 10 \mathrm{~mW} / \mathrm{cm}^{2}$. The band-edge emission at $295 \mathrm{~K}$ has a width of $50 \mathrm{meV}$ and is much stronger than the yellow deep-level luminescence. The intensity oscillations with photon energy result from a Fabry-Perot cavity formed by the GaN/HfN and GaN/air interfaces. ${ }^{20}$ The large amplitude of the oscillations attests to the high interface quality. At $11 \mathrm{~K}$ the donor-bound exciton transition appears at $3.455 \mathrm{eV}$ along with its phonon replica at $3.360 \mathrm{eV}$. Comparison with the value of $3.471 \mathrm{eV}$ for strain-free $\mathrm{GaN}$ gives a $0.54 \mathrm{GPa}$ tensile stress in the epilayer. ${ }^{21,22}$ This is attributed to thermal mismatch with the Si substrate and possibly the small lattice mismatch with the HfN buffer layer. The inhomogenous broadening of the peak suggests nonuniform strain. The peak position and full-width at half-maximum $(17 \mathrm{meV})$ are comparable to the best results reported for GaN on AlN/Si(111) using a single buffer layer process. ${ }^{1,2,7}$ A peak width as narrow as 5$6 \mathrm{meV}$ has been reported for layers grown on AlGaN/GaN superlattices. ${ }^{3,5}$

Although most GaN growth efforts on Si focused on the (111) surface due to its hexagonal symmetry, $\mathrm{Si}(001)$ is more commonly used in microelectronics. GaN grown on $\mathrm{Si}(001)$ often results in a mixture of wurtzite- and zincblende-structure grains. ${ }^{23} \mathrm{In}$ contrast, $\mathrm{x}$-ray $\theta-2 \theta$ scans for layers on $\mathrm{HfN} / \mathrm{Si}(001)$ indicated pure wurtzite $\mathrm{GaN}$ with a single crystallite orientation in both the [0002] and [10-11] directions. The GaN(0002) plane was confirmed to be parallel to $\mathrm{Si}(004)$, but the in-plane orientation relationship has yet to be determined. The (0002) rocking curve width was 37 arcmin, and the intensity of the (10-11) reflection was too low to obtain a meaningful rocking curve width. The reduced structural quality is not surprising, since $\operatorname{HfN}(111)$ provides a hexagonal template for $\mathrm{GaN}$ nucleation while $\mathrm{HfN}(001)$ does not. 
A clearly different luminescence spectrum is observed for $\mathrm{GaN}$ on the (001) substrate orientation, as shown in Fig. 2. Compared with Fig. 1 the donor-acceptor pair transition is more prominent and the band-edge peak is broader. However, band-edge peak width of $72 \mathrm{meV}$ at $295 \mathrm{~K}$ achieved for the epilayer on $\mathrm{HfN} / \mathrm{Si}(001)$ compares favorably to the $82 \mathrm{meV}$ reported for $\mathrm{GaN}$ on $\mathrm{AlN} / \mathrm{Si}(001) .{ }^{4}$ The integrated luminescence intensities in Figs. 1 and 2 are equal within experimental error for all measurement temperatures. Thus it is concluded that although $\mathrm{HfN} / \mathrm{Si}(001)$ results in markedly worse GaN x-ray rocking curves than $\mathrm{HfN} / \mathrm{Si}(111)$, the levels of non-radiative recombination for the two substrate orientations are comparable.

The most striking difference in the luminescence spectra for $\mathrm{GaN}$ films on the HfN(111) and (001) orientations is in the near-edge spectrum. While Fig. 1 shows a single donor-bound exciton line at low temperature, in Fig. 2 two broad peaks are observed at 3.465 and $3.407 \mathrm{eV}$, the former of which is resolved into narrow but overlapping peaks at 3.471 and $3.461 \mathrm{eV}$. The highest energy transition is assigned to the donor-bound exciton, while the others have yet to be identified conclusively. A peak often observed at 3.40-3.42 eV has been attributed to an exciton bound to a stacking fault. ${ }^{24}$ A high density of stacking faults might indeed be expected for wurtzite GaN grown on a cubic template.

From the considerably higher energy of the band-edge emission in Fig. 2 vs. Fig. 1 it is concluded that the tensile stress in $\mathrm{GaN}$ on $\mathrm{HfN}(001)$ is much less than that on HfN(111). The difference could be related to structural defects in the epilayer on $\mathrm{HfN}(001)$ that impede transfer of thermal strain from the substrate. The negligible strain in the $1.2 \mu \mathrm{m}$ film suggests that crack-free GaN much thicker than $1.2 \mu \mathrm{m}$ can be grown 
on this substrate orientation. Additional work is needed to determine the GaN maximum crack-free thickness and to understand the reason for the very low stress on $\mathrm{HfN} / \mathrm{Si}(001)$. Wan et al. $^{4}$ also observed low stress in crack-free $2 \mu \mathrm{m}$ wurtzite GaN films on AlN/Si(001), suggesting that the substrate orientation may be the key factor rather than the HfN buffer layer material.

$\mathrm{HfN}(001)$ is in principle an interesting template for zincblende $\mathrm{GaN}$ due to its cubic symmetry and near-exact lattice match. Synthesis of the metastable zincblende phase on GaAs and 3C-SiC substrates has only been achieved for a limited growth parameter space involving the substrate temperature, $\mathrm{Ga} / \mathrm{N}$ ratio, and deposition rate. ${ }^{25}$ The substrate temperature and growth rate in the present experiments were significantly higher than those successfully used for zincblende GaN growth. This might explain the fact that wurtzite rather than zincblende material was obtained on $\mathrm{HfN}(001)$. It is possible, however, that the small grain size of the present HfN films would hinder nucleation of zincblende GaN even for ideal growth conditions. An additional range of growth parameters involving lower substrate temperatures and lower deposition rates need to be investigated to determine the feasibility of $\mathrm{HfN}(001)$ as a template for zincblende GaN.

In conclusion, it was shown that $\mathrm{HfN}$ is an effective buffer layer for epitaxy of wurtzite $\mathrm{GaN}$ on $\mathrm{Si}$. The maximum crack-free thickness obtained was $1.0 \mu \mathrm{m}$ on $\mathrm{Si}(111)$ and $1.2 \mu \mathrm{m}$ on $\mathrm{Si}(001)$. The $\mathrm{HfN} / \mathrm{Si}(111)$ substrates yielded narrower $\mathrm{GaN}$ x-ray rocking curves and sharper luminescence peaks, while epilayers grown on $\mathrm{HfN} / \mathrm{Si}(001)$ exhibited much lower residual strain with equal integrated luminescence intensity. The GaN properties obtained thus far without yet optimizing the HfN deposition temperature are 
comparable to typical results achieved with AlN buffer layers. The superior diffusion resistance, closer lattice match, and metallic conductivity of HfN are advantages over AlN buffer layers for certain applications. While the present results were achieved by MBE, we expect that HfN buffer layers would also be effective for MOVPE growth of GaN on Si.

This work was supported by the Director, Office of Science, Office of Basic Energy Sciences, Division of Materials Sciences, of the U.S. Department of Energy under contract No. DE-AC03-76SF00098. J.G. was supported by the Alexander von Humboldt Association through a F. Lynen fellowship. Thanks are also due to GAN Semiconductor, Inc. for providing the rf plasma source, and to Dr. Mike Rubin for use of laboratory equipment.

\section{REFERENCES}

1 M.A. Sánchez-García, F.B. Naranjo, J.L. Pau, A. Jiménez, E. Calleja, E. Muñoz, S.I. Molina, A.M. Sánchez, F.J. Pacheco, and R. García, Phys. Stat. Sol. A 176, 447 (1999).

2 F. Semond, B. Damilano, S. Vézian, N. Grandjean, M. Leroux, and J. Massies, Phys. Stat. Sol. B 216, 101 (1999).

3 S.A. Nikishin, N.N. Faleev, V.G. Antipov, S. Francoeur, L. Grave de Peralta, G.A. Seryogin, H. Temkin, T.I. Prokofyeva, M. Holtz, and S.N.G. Chu, Appl. Phys. Lett. 75, 2073 (1999).

4 J. Wan, R. Venugopal, M.R. Melloch, H.M. Liaw, and W.J. Rummel, Appl. Phys. Lett. 79, 1459 (2001). 
5 E. Feltin, B. Beaumont, M. Laügt, P. de Mierry, P. Vennéguès, H. Lahrèche, M. Leroux, and P. Gibart, Appl. Phys. Lett. 79, 3230 (2001).

6 H. Marchand, L. Zhao, N. Zhang, B. Moran, R. Coffie, U. K. Mishra, J. S. Speck, S. P. DenBaars, and J.A. Freitas, J. Appl. Phys. 89, 7846 (2001).

7 A. Strittmatter, A. Krost, J. Bläsing, and D. Bimberg, Phys. Stat. Sol. A 176, 611 (1999).

8 C.I. Park, J.H. Kang, K.C. Kim, K.S. Nahm, E.-K. Suh, and K.Y. Lim, Thin Solid Films 401, 60 (2001).

9 D. Wang, Y. Hiroyama, M. Tamura, M. Ichikawa, and S. Yoshida, Appl. Phys. Lett. 77, 1846 (2000).

10 N. Kobayashi, J. Kobayashi, W.-J. Choi, P.D. Dapkus, X. Zhang, and D.H. Rich, J. Cryst. Growth 189/190, 172 (1998).

11 T. Yodo, H. Ando, D. Nosei, J. Seko, K. Sakai, M. Shimeno, and Y. Harada, J. Cryst. Growth 233, 22 (2001).

12 S. Nishimura, S. Matsumoto, and K. Terashima, Optical Materials 19, 223 (2002).

13 B.J. Zhang, T. Egawa, H. Ishikawa, N. Nishikawa, T. Jimbo, and M. Umeno, Phys. Stat. Sol. A 188, 151 (2001).

14 A. Dadgar, J. Christen, T. Riemann, S. Richter, J. Bläsing, A Diez, A. Krost, A. Alam, and M. Heuken, Appl. Phys. Lett. 78, 2211 (2001).

15 C.A. Tran, A. Osinski, R.F. Karlicek, Jr., and I. Berishev, Appl. Phys. Lett. 75, 1494 (1999).

16 E. Calleja, M.A. Sánchez-García, D. Basak, F.J. Sánchez, F. Calle, P. Youinou, E. Muñoz, J.J. Serrano, J.M. Blanco, C. Villar, T. Laine, J. Oila, K. Saarinen, P. 
Hautojärvi, C.H. Molloy, D.J. Somerford, and I. Harrison, Phys. Rev. B 58, 1550 (1998).

17 S. Shinkai and K. Sasaki, Jpn. J. Appl. Phys. Part 1 38, 2097 (1999).

18 Q.Z. Liu and S.S. Lau, Sol. State Electron. 42, 675 (1998).

19 B. Heying, X.H. Wu, S. Keller, Y. Li, D. Kapolnek, B.P. Keller, S.P. DenBaars, and J.S. Speck, Appl. Phys. Lett. 68, 643 (1996).

20 A. Billeb, W. Grieshaber, D. Stocker, E.F. Schubert, and R.F. Karlicek Jr., Appl. Phys. Lett. 70, 2790 (1997).

21 K. Kornitzer, T. Ebner, K. Thonke, R. Sauer, C. Kirchner, V. Schwegler, M. Kamp, M. Leszczynski, I. Grzegory, and S. Porowski, Phys. Rev. B 60, 1471 (1999).

22 C. Kisielowski, J. Krüger, S. Ruvimov, T. Suski, J.W. Ager III, E. Jones, Z. Liliental-Weber, H. Fujii, M. Rubin, E.R. Weber, M.D. Bremser, and R.F. Davis, Phys. Rev. B 54, 17745 (1996).

23 B. Yang, A. Trampert, O. Brandt, B. Jenichen, and K.H. Ploog, J. Appl. Phys. 83, 3800 (1998).

24 Y.T. Rebane, Y.G. Shreter, and M. Albrecht, Phys. Stat. Sol. A 164, 141 (1997).

25 S. Yoshida, Physica E 7, 907 (2000). 


\section{Figure Captions Armitage et al. Applied Physics Letters}

Figure 1. Photoluminescence spectra for an intact region of a $1.5 \mu \mathrm{m}$ cracked GaN epilayer on $\mathrm{HfN} / \mathrm{Si}(111)$. The weak yellow luminescence in the range $2.0-2.5 \mathrm{eV}$ and donor-acceptor pair luminescence from $2.8-3.2 \mathrm{eV}$ are strongly modulated by interference effects.

Figure 2. Photoluminescence spectra for a $1.2 \mu \mathrm{m} \mathrm{GaN}$ epilayer on $\mathrm{HfN} / \mathrm{Si}(001)$. The Fabry-Perot cavity formed by the epilayer results in strong modulation of the luminescence. 
Figure 1.

Armitage et al. Applied Physics Letters

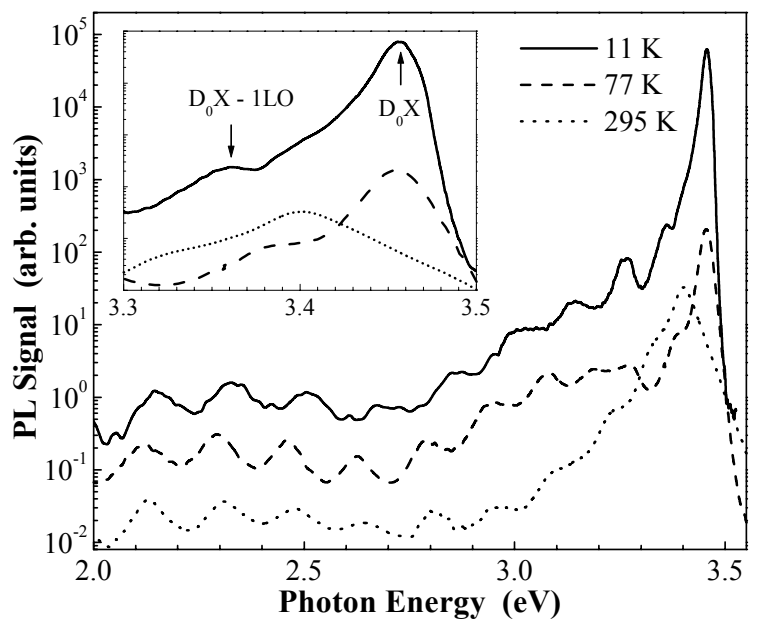


Figure 2.

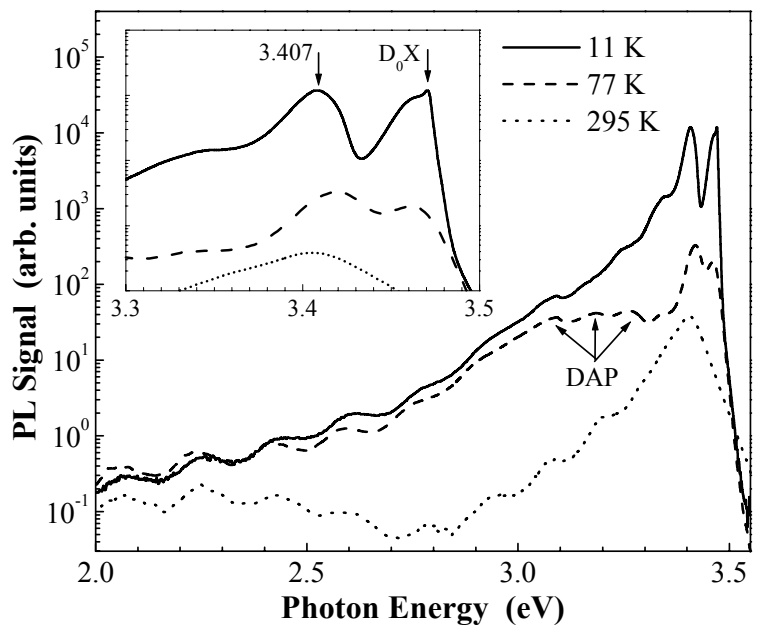

